
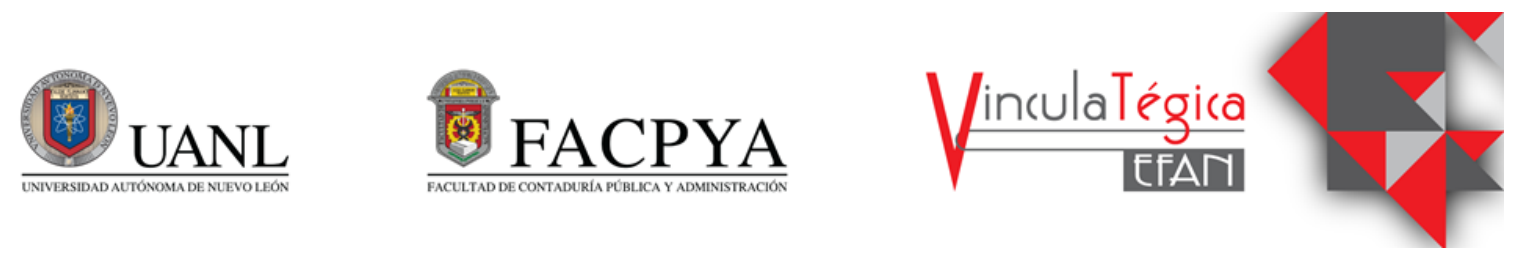

\title{
Análisis socioespacial de la vivienda ante Covid-19 en la Zona Metropolitana de Monterrey
}

\author{
Jazmín Flores Guevara ${ }^{1}$ y Ana Elizabeth Flores Marín ${ }^{2}$ \\ ${ }^{1}$ Universidad Autónoma de Nuevo León, jazmin.floresgvr@uanl.edu.mx, Ciudad Universitaria, San Nicolás \\ de los Garza, Nuevo León, México. C.P. 66451, 8132698846 \\ ${ }^{2}$ Universidad Autónoma de Nuevo León, ana.floresmrn@uanl.edu.mx, Ciudad Universitaria, San Nicolás de \\ los Garza, Nuevo León, México. C.P. 66451, 8110051683
}

Información del artículo revisado por pares

Fecha de aceptación: junio-2021

Fecha de publicación en línea: diciembre-2021

DOI: https://doi.org/10.29105/vtga7.1-143

\section{Resumen}

Este artículo presenta una visualización de la conducta de los habitantes de la zona metropolitana de Monterrey, Nuevo León, México y las adecuaciones que han realizado en sus viviendas a raíz de la pandemia por Covid-19. Se efectúa un análisis de estadísticos descriptivos, frecuencias, tablas de contingencia y análisis de correlación. Con la finalidad de conocer la versatilidad de los espacios en la vivienda y cómo los habitantes de la Zona Metropolitana de Monterrey se han visto en la necesidad de realizar modificaciones en sus hábitos y estilo de vida. Los participantes del estudio fueron 547 habitantes de la zona metropolitana de Monterrey, los resultados muestran que los habitantes de los municipios más cercanos a la capital son los que perciben una adaptación personal y de su vivienda con mayor nivel. Los encuestados con menor conocimiento de casos de Covid-19 son aquellos que expresaron una mayor percepción de adaptación personal a diferencia de quienes indicaron estar o haber estado enfermo con un nivel menor. La recámara resultó el espacio predilecto por los encuestados al ser más usado para trabajo y ocio. Entre las principales adecuaciones se encontraron los cambios estéticos por los encuestados del género masculino y plantar vegetación por el género femenino.

Palabras clave: conducta, adecuación, vivienda, Covid-19, estadísticos descriptivos.

\begin{abstract}
This article present a visualization of the human behavior of the citizens of the metropolitan area of Monterrey, Nuevo León, México and the changes in the household as a consequence of Covid-19. This article includes an analysis of descriptive statistics, frequency and crosstabs, analysis of correlation. With the aim of knowing the possibilities of changes and versatility of spaces in the living place and how the citizens of the metropolitan area of Monterrey have had the necessity of making modifications in their habits and general routines. The study has the recollection of 547 citizens of the metropolitan area of Monterrey, the results show that de cities near the capital are more adapted in an individual way and their living places. The surveyed with less knowledge of Covid-19 cases are those who expressed a greater perception of personal adaptation, as opposed to those who indicate being or having been sick with a lower level. The bedroom was the prefered space for those surveyed as it was most used for work and leisure. Among the main adjustments were aesthetic changes by male respondents and planting vegetation by female gender.
\end{abstract}

Keywords: human behavior, redesign, household, Covid-19, descriptive statistics.

JEL: Z00, Z10, Z13. 


\section{INTRODUCCIÓN}

El Covid-19 coronavirus disease es la enfermedad causada por el nuevo coronavirus conocido como SARS-CoV-2. La Organización Mundial de la Salud (OMS) tuvo noticia por primera vez de la existencia de este nuevo virus el 31 de diciembre de 2019, al ser informada de un grupo de casos de «neumonía vírica» que se habían declarado en Wuhan en la República Popular China. El 11 de marzo de 2020 la OMS declaró el Covid-19 como pandemia. Se le llama pandemia a la propagación mundial de una nueva enfermedad. (Organización Mundial de la Salud, 2020).

De acuerdo con el informe de situación 65 de la OMS, con fecha del 25 de marzo de 2020, en el mundo se habían reportado 414,179 casos confirmados y 18,440 muertes (OMS,2020). Acorde a la cifra más actual registrada con fecha 2 de marzo de 2021, más de 2.6millones de nuevos casos fueron registrados la última semana del mes de febrero, un aumento del $7 \%$ en comparación con la cifra anterior registrada. (OMS, 2021). Las cifras continúan siendo alarmantes, a más de un año del comienzo de la pandemia, la evidente falta de control de la expansión del Covid-19 ha tenido como consecuencia una crisis global donde la economía y la salud de la sociedad han sido fuertemente afectadas.

La pandemia por Covid-19 ha tenido un gran impacto en todo el mundo, comenzando por la medida de vivir en confinamiento. Pasar todo o gran parte del tiempo en casa y realizar acciones de trabajo o escuela en ella ha dejado como resultado la creación de nuevos hábitos, entre ellos, guardar la distancia, evitar espacios con aglomeraciones, lavarnos las manos con mayor frecuencia, usar desinfectante y el uso del cubrebocas. Aunado a estos nuevos hábitos, ha surgido la necesidad por modificar y adecuar los espacios en los que nos desarrollamos, cambiando las formas de habitar e incluso deshabitar espacios de ocio y entretenimiento.

La vivienda, por su parte, es la tipología más importante de la sociedad, en ella nos desarrollamos y encontramos el hogar. Además, esta pandemia no solo les ha mostrado a los gobiernos lo deficiente que se encontraban sus sistemas de salud, lo frágil que eran sus economías y los problemas políticos que podrían pasar sus naciones, sino que dio a conocer como la mayoría de las viviendas no cumplían con los requisitos para que las personas logren habitar, descansar y recrearse en ellas, ni siquiera la movilidad ni el transporte, que son pilares de la sostenibilidad urbana, están excluidos de esta problemática. (García, 2017) El confinamiento ha propiciado la creación y remodelación de espacios que a principios del año 2020 no considerábamos como esenciales e incluso eran nulos dentro de la vivienda. A pesar de que las nuevas tipologías arquitectónicas de la vivienda buscan integrar la mayor cantidad de usos en ella, es claro que la arquitectura que está por implementarse en la situación post Covid-19 buscará ser mayormente autosuficiente, en donde las dinámicas de habitar buscarán facilitar todas las actividades humanas en un solo lugar.

Por todos aquellos factores previamente mencionados la vivienda ha sido el refugio y el ente más importante en la ciudad para controlar el virus, mientras la vacuna o alguna otra forma médica de combatirlos esté al alcance de toda la sociedad, la vivienda juega un papel fundamental de protección y prevención ante el Covid-19, por ello es indispensable que se cuenten con las condiciones óptimas que colaboren en la reducción del contagio.

En la zona metropolitana de Monterrey los estudios sobre el impacto del Covid-19 en la vivienda y cómo las adecuaciones de esta han influido en la forma de vivir de las personas son prácticamente nulos, de ahí el interés por contestar la siguiente pregunta de investigación, ¿cómo los habitantes de la zona metropolitana de Monterrey han adaptado y adecuado su vivienda para vivir en confinamiento a raíz de la pandemia por Covid-19?

En el caso de este artículo se aplica un análisis de los estadísticos descriptivos, de frecuencia, tablas de contingencia y un análisis de correlación para identificar los 
espacios más versátiles en la vivienda, la satisfacción las personas con sus viviendas en torno al nuevo estilo de vida en confinamiento y los cambios más frecuentes que se han presentado en la vivienda.

Con el objetivo de conocer los efectos del COVID-19 se centró el interés en analizar a los habitantes de la zona metropolitana de Monterrey y la manera en que se han adaptado a vivir en confinamiento adecuando sus viviendas. Obteniendo resultados en dos panoramas que enfrentan los individuos, primero, aquellos que se han visto envueltos en la necesidad de modificar su rutina diaria y los espacios donde habitan y segundo, aquellos que continúan con la rutina de manera habitual y saliendo de casa. De esta manera buscamos aportar información sobre el impacto del COVID-19 en las viviendas y los habitantes de la zona metropolitana de Monterrey durante el confinamiento.

El trabajo se organiza de la siguiente manera. En la sección de metodología se describe la obtención de los datos de cada uno de los constructos desarrollados, datos sociodemográficos, COVID-19, trabajo en casa, ocio en casa, espacios exteriores en casa y percepción ante COVID-19; en la sección de resultado, el método de análisis empleado, su descripción, además aparecen los hallazgos obtenidos con el instrumento y, por último, se presentan algunas conclusiones apoyadas del trabajo realizado.

\section{MARCO TEÓRICO}

La pandemia por COVID-19 ha resaltado la importancia del entorno y el desarrollo de las ciudades en el ámbito central de la vivienda, este fenómeno nos ha hecho profundizar y concientizar lo bien que le hemos hecho a la naturaleza ante el confinamiento que hemos vivido, recordando el objetivo 11 planteado en la Agenda 2030 de la Organización de las Naciones Unidas, lograr que las ciudades y los asentamientos humanos sean inclusivos, seguros, resilientes y sostenibles.

En América Latina, el $21 \%$ de la población urbana vive en barrios marginales, asentamientos informales $o$ viviendas inadecuadas, siendo su población urbana el $81 \%$ del total. (Universidad Politécnica de
Madrid, 2020). Es en este segmento de la población en donde la pandemia ha repercutido con mayor impacto, sin embargo, es también este sector el que se ha visto mayormente orillado a implementar adecuaciones en la vivienda debido a su alta exposición con el virus y la modificación en el estilo de vida.

\subsection{COVID-19}

La pandemia por COVID-19 ha remarcado la brecha social que existe en nuestro país, afectado en gran medida a las clases socioeconómicas media y baja como el sector más vulnerable ante la situación.

En México y el resto de Latinoamérica la débil protección social también obliga a muchas personas mayores a continuar trabajando fuera de casa, a menudo en ocupaciones de baja calificación y en condiciones de informalidad. Si no es el caso, la corresidencia con personas más jóvenes y otros hogares las pone frecuentemente en contacto con quienes sí deben y necesitan salir a trabajar. Pautas complejas de corresidencia están asociadas a situaciones laborales del hogar más precarias $\mathrm{y}$ mayores dificultades para minimizar el contacto social (Módenes, 2020).

\subsection{Vivienda en COVID-19}

Una vivienda adecuada garantiza la mejora continua de las condiciones de vida de todas las personas y el disfrute de otros derechos económicos, sociales y culturales. La Constitución Política de México establece, en su artículo $1^{\circ}$, el compromiso del Estado de lograr la protección más amplia para todas las personas en el campo de los derechos humanos, y en su artículo $4^{\circ}$ reconoce el derecho de toda familia a una vivienda digna y decorosa (Programa de las Naciones Unidas para los Asentamientos Humanos ONUHábitat, 2018). Sin embargo, la pandemia de COVID-19 ha resaltado las carencias y precariedad de la vivienda en México y de la falta de inferencia del Estado ante la situación global y ante este derecho fundamental de la sociedad. 
La vivienda, particularmente la de interés social, constituye uno de los ejes más importantes en la planificación urbana; una vivienda adecuadamente diseñada en función de las características, necesidades y expectativas de los usuarios, su entorno y la relación con la ciudad, resulta esencial para el desarrollo psicológico y social, favorece la sustentabilidad urbana y contribuye a elevar el bienestar con un menor costo futuro, reduciendo a la vez el impacto ambiental (Moreno y Alvarado, 2011). Sin embargo, los modelos para la gestión de la vivienda de interés social que han predominado en América Latina durante las últimas décadas generan soluciones orientadas hacia los aspectos cuantitativos, mientras que la calidad, y particularmente la del diseño, es subvalorada. El diseño de la vivienda de interés social deberá ser flexible, permitiendo la adecuación de la vivienda de forma progresiva, y que evolucione en el tiempo gracias a su sostenibilidad económica como una vivienda productiva, involucrando a su vez la participación del usuario como actor principal de la mano con el profesional de la arquitectura (Pérez, 2015).

La pandemia por COVID-19 ha creado retos sociales para la implementación de nuevas dinámicas en casa, impulsando a los grupos más vulnerables a la adaptación de espacios y modificación del estilo de vida.

\subsection{La vida post COVID-19}

ONU-Hábitat, el programa de las Naciones Unidas para los asentamientos humanos presentó el informe 'Ciudades y pandemias: hacia un futuro más justo, verde y equitativo'; el cual demuestra cómo las ciudades pueden reducir el impacto de futuras pandemias y ser más prósperas, justas y respetuosas con el medio ambiente. Asimismo, el informe describe cómo puede surgir una nueva normalidad en las ciudades donde la salud, la vivienda y la seguridad se priorizan para los más vulnerables no solo por necesidad social, sino también por un profundo compromiso con los derechos humanos para todos. Esto requiere que los gobiernos se concentren en políticas para proteger los derechos sobre el suelo, mejorar el acceso al agua, el saneamiento, el transporte público, la electricidad, las instalaciones de salud y educación y garantizar una conectividad digital inclusiva. (ONU-Hábitat, 2021)

Se espera que el futuro de la vivienda logre ser flexible y multifuncional, de manera que la forma de habitar sea versátil y digna. Este enfoque es una oportunidad para que el Estado se involucre y haga de la ciudad un lugar mejor en conjunto con la sociedad.

Debido a la problemática que acontece ante la vivienda de forma global y la sociedad en general surge el interés del análisis presentado en este trabajo siendo que los estudios sobre el impacto del COVID-19 en la vivienda continúan siendo escasos en la zona metropolitana de Monterrey. Por ello se enfoca en conocer la manera en que los ciudadanos han optado por adecuar sus viviendas y adaptarse a un nuevo esquema de vida.

\section{MÉTODO}

Estudiar a los habitantes de la zona metropolitana de Monterrey y como han adaptado su vivienda durante el COVID-19 ha sido un reto por la variedad de estilos de vida de los habitantes, así como por los numerosos factores socioeconómicos que pueden condicionar dichos cambios. Para determinar la manera en que los habitantes de la zona metropolitana de Monterrey han adaptado su vivienda y el comportamiento de éstos, diseñamos una encuesta aplicada a personas mayores de 16 años, durante el cuarto trimestre del año 2020. Los entrevistados se eligieron por su disposición a responder y por este motivo no se distribuyeron de manera uniforme en los diferentes municipios de la zona metropolitana de Monterrey.

Realizamos un muestreo no probabilístico; utilizando como instrumento una encuesta digital con la herramienta de Google Forms, que nos dio la posibilidad de llegar a más personas sin costo alguno y permitiendo compartir el link por medio de redes sociales personales como Instagram, Facebook y WhatsApp. El muestreo probabilístico es una técnica que consiste en una investigación realizada sobre una muestra de sujetos utilizando procedimientos 
estandarizados de interrogación con el fin de conseguir mediciones cuantitativas sobre una cantidad de características de la población (García, 1992).

Con base a la fórmula para calcular el tamaño de una muestra de una población finita de variable cualitativa (Murray \& Larry, 2009).

$$
\begin{gathered}
n=\frac{N * Z^{2} * p * q}{e^{2} *(N-1)+Z^{2} * p * q} \\
n=\frac{4,899,119 * 1.96^{2} * 0.50 * 0.50}{0.05^{2} *(4,899,119-1)+1.96^{2} * 0.50 * 0.50} \\
n=384.129 \approx 385
\end{gathered}
$$

Dónde $\mathrm{N}$ es la población de la zona metropolitana de Monterrey de 4,899,119, (INEGI, 2020), $\mathrm{Z}$ es igual al nivel de confianza 1.96 (Nivel de confianza del 95\%), p siendo la probabilidad de que ocurra el evento estudiado con un 50\%. q siendo la con un $50 \%$ de que no ocurra el evento y con margen de error del 5\%. Por lo que el tamaño de la muestra ideal mínimo para el estudio fue de 385 personas, logrando recolectar una muestra mayor.

Obteniendo un total de 547 encuestas recolectadas de manera digital. El cuestionario que se aplicó está compuesto por 22 preguntas organizadas en seis bloques, en el primero se hizo referencia a las características sociodemográficas (género, edad, estado civil, grado escolar, ocupación, municipio en el que habita, número de integrantes que habitan la vivienda, etc.), mientras que en el segundo se enfocó al conocimiento de casos de COVID-19 en la zona, actividades y frecuencia de salidas de los sujetos de estudio. En el tercer y cuarto bloque analizamos los espacios del hogar que se han adaptado y el tipo de adecuación que se realizó (cambios estéticos, constructivos, etc.), además de las adecuaciones realizadas en espacios exteriores analizadas en el quinto bloque y por último se cuestionó sobre la percepción de los habitantes de la zona metropolitana de Monterrey sobre el nivel de adaptación ante el confinamiento.

Debido a la cantidad de datos que se manejan y la variedad de análisis que se realizan se rebasa la capacidad del cálculo manual y se han empleado herramientas como el SPSS que junto con el BMDP son los más utilizados en investigación aplicada a las Ciencias Sociales (Bisquerra, 1989). Es por eso que partir de esta información, la herramienta de análisis que empleamos en este proyecto fue el Statistical Package for the Social Sciences (SPSS) para la realización de análisis de estadísticos descriptivos, de frecuencias, tablas de contingencia y análisis de correlación que nos permitirá abordar la complejidad del tema, con la finalidad de probar la hipótesis de que los espacios de la vivienda han sido adaptados mediante adecuaciones y continuarán presentándose de manera que el comportamiento humano evolucione, resurja y se reinvente tras la enfermedad de COVID-19.

\section{RESULTADOS}

Del instrumento se obtuvo una muestra total de 547 encuestados, variables que se encuentran descritas en la siguiente Tabla 1. Las variables buscan identificar la manera en que los habitantes han adaptado su hogar y estilo de vida durante el confinamiento. A partir del segundo párrafo utilizar sangría en la primera línea.

Tabla 1. Descripción de variables.

\begin{tabular}{ll}
\hline \multicolumn{1}{c}{ Variable } & \multicolumn{1}{c}{ Descripción } \\
\hline Género & Género por el cual se identifican los sujetos de estudio 1=masculino,2= femenino, 3= otro. \\
Edad & Muestra la edad del encuestado en años cumplidos de forma numérica. \\
Estado_civil & Estado del sujeto de estudio con respecto a su condición de pareja. \\
Estudios & Nivel de estudios completados. \\
Ocupación & Actividad a la que se dedican los sujetos de estudio. \\
Municipio & Municipio de la zona metropolitana de Monterrey en donde habitan los sujetos de estudio. \\
Vivienda & Tipo de vivienda donde habitan los sujetos de estudios.
\end{tabular}


$\begin{array}{ll}\text { Habitantes } & \text { Número de habitantes con los que comparten vivienda los sujetos de estudio. } \\ \text { COVID-19 } & \text { Conocimiento sobre casos de COVID-19 en el entorno del sujeto de estudio. } \\ \text { Incomodidad } & \text { Actividades que le causan mayor incomodidad a los sujetos de estudio. } \\ \text { Frecuencia_sem } & \text { Frecuencia con lo que sale de la vivienda hoy en día los sujetos de estudio. } \\ \text { Visitas } & \text { Lugares que más visita fuera de casa hoy en día. } \\ \text { Espacio_trabajo } & \text { Espacio que se adecuó en la vivienda para trabajo y escuela. } \\ \text { Ade_trabajo } & \text { Adecuaciones que realizaron los sujetos de estudio para espacio de trabajo y escuela. } \\ \text { Espacio_ocio } & \text { Espacio que se adecuó en la vivienda para el acceso, ocio y entretenimiento. } \\ \text { Ade_ocio } & \text { Adecuaciones que realizaron los sujetos de estudio para espacio de ocio y entretenimiento. } \\ \text { Exteriores } & \text { Espacios exteriores con los que ya contaba la vivienda antes de COVID-19. } \\ \text { Ade_exteriores } & \text { Adecuaciones que realizaron los encuestados en espacios exteriores. } \\ \text { Espacio favorito } & \text { Espacio favorito dentro de la vivienda de los sujetos de estudio. } \\ \text { Adap_personal } & \text { Nivel de adaptación personal de los sujetos de estudio ante vivir en confinamiento por } \\ & \text { COVID-19. 1=Nada, 2=Poco, 3=Neutro, 4=Suficiente, 5=Mucho. } \\ \text { Adap_vivienda } & \text { Nivel de adaptación de la vivienda de los sujetos de estudio ante vivir en confinamiento } \\ & \text { por COVID-19. 1=Nada, 2=Poco, 3=Neutro, 4=Suficiente, 5=Mucho. }\end{array}$ Fuente: Elaboración propia

En la siguiente Tabla 2 aparecen los estadísticos descriptivos que analizan a la muestra que tiene como cualidad que todos los encuestados son habitantes de la zona metropolitana de Monterrey ante la pandemia de COVID-19. Se observa en la variable edad, los años cumplidos de forma numérica, con un promedio de 29.70 años en los encuestados, así mismo la variable habitantes, representa el número de personas con los que comparten vivienda los sujetos de estudio, con una media de 4.01 habitantes por vivienda, mientras que en las variables de percepción ante la adaptación personal y la adaptación de la vivienda fueron medidas en una escala con valoración máxima de 5 , resultando una media de 3.68 en la adaptación personal y 3.62 en la adaptación de la vivienda, por lo que se infiere que la muestra percibe una adaptación neutral acercándose a ser suficiente.

Tabla 2. Estadísticos descriptivos

\begin{tabular}{lrrrr}
\hline Variable & Mínimo & \multicolumn{1}{c}{ Máximo } & Media & Desviación \\
\hline Género & 1 & 2 & 1.64 & 0.481 \\
Edad & 16 & 72 & 29.70 & 14.219 \\
Estado_civil & 1 & 5 & 1.44 & 0.857 \\
Estudios & 1 & 8 & 4.37 & 1.241 \\
Ocupación & 1 & 9 & 4.15 & 2.759 \\
Municipio & 1 & 12 & 6.10 & 2.800 \\
Vivienda & 1 & 4 & 1.27 & 0.623 \\
Habitantes & 1 & 11 & 4.01 & 1.489 \\
covid-19 & 1 & 4 & 2.95 & 0.527 \\
Incomodidad & 1 & 4 & 2.53 & 1.073 \\
Frecuencia_sem & 1 & 3 & 1.52 & 0.747 \\
Espacio_Trabajo & 1 & 6 & 3.48 & 1.522 \\
Ade_Trabajo & 1 & 3 & 2.22 & 0.959 \\
Espacio_Ocio & 1 & 6 & 3.46 & 1.587 \\
Ade_Ocio & 1 & 3 & 2.34 & 0.927
\end{tabular}




\begin{tabular}{lrrrr} 
Espacio favorito & 1 & 5 & 3.59 & 1.578 \\
Adap_Personal & 1 & 5 & 3.68 & 1.034 \\
Adap_vivienda & 1 & 5 & 3.62 & 1.106 \\
Total & 547 & & & \\
\hline Fuente: Elaboración propia & &
\end{tabular}

Para lograr el objetivo de la investigación y conocer la manera en que hemos ido adaptando nuestra vivienda y nuestras actividades ante la nueva normalidad, fue necesario asociar las variables de nuestra herramienta de investigación, primeramente variables que midan las características sociodemográficas de la población, realizando un análisis de estadísticos de frecuencias donde se observa que la muestra está integrada por 349 encuestados del género femenino, el $63.8 \%$ y 198 identificados con el género masculino, $36.2 \%$ de la muestra, con un rango de edad predominante entre los 18 y 24 años de edad, en su mayoría solteros con el $69.7 \%$, y siendo estudiantes el $35.8 \%$ la ocupación con más frecuencia entre los encuestados, el $26.7 \%$ son residentes del municipio de Monterrey, además el $81.2 \%$ de los encuestados habitan en una vivienda unifamiliar siendo esta tipología de vivienda la predominante como se muestra la Tabla 3.

Tabla 3. Estadísticos de frecuencias

\begin{tabular}{|c|c|c|}
\hline Género & Frecuencia & Porcentaje \\
\hline Femenino & 349 & 63.8 \\
\hline Masculino & 198 & 36.2 \\
\hline Total & 547 & 100 \\
\hline Edad & Frecuencia & Porcentaje \\
\hline $15-17$ & 57 & 10.4 \\
\hline $18-24$ & 279 & 51 \\
\hline $25-40$ & 94 & 17.2 \\
\hline $41-60$ & 91 & 16.6 \\
\hline 61 o más & 26 & 4.8 \\
\hline Total & 547 & 100 \\
\hline Estado_civil & Frecuencia & Porcentaje \\
\hline Soltero & 381 & 69.7 \\
\hline Casado & 130 & 23.8 \\
\hline Divorciado & 10 & 1.8 \\
\hline Viudo & 11 & 2 \\
\hline Unión libre & 15 & 2.7 \\
\hline Total & 547 & 100 \\
\hline Ocupación & Frecuencia & Porcentaje \\
\hline Estudiante & 196 & 35.8 \\
\hline Técnico & 5 & 0.9 \\
\hline Trabajador de oficio & 8 & 1.5 \\
\hline Trabajador/ empleado & 123 & 22.5 \\
\hline Comerciante/ vendedor & 5 & 0.9 \\
\hline Emprendedor/ negocio propio & 35 & 6.4 \\
\hline Profesionista & 112 & 20.5 \\
\hline Ama/o de casa/ me dedico al hogar & 39 & 7.1 \\
\hline Desempleado & 24 & 4.4 \\
\hline Total & 547 & 100 \\
\hline Municipio & Frecuencia & Porcentaje \\
\hline Apodaca & 77 & 14.1 \\
\hline Cadereyta Jiménez & 2 & 0.4 \\
\hline García & 6 & 1.1 \\
\hline General Escobedo & 68 & 12.4 \\
\hline
\end{tabular}




\begin{tabular}{lrr} 
Guadalupe & 72 & 13.2 \\
Juárez & 14 & 2.6 \\
Monterrey & 146 & 26.7 \\
Salinas Victoria & 2 & 0.4 \\
San Nicolás de los Garza & 126 & 23 \\
Santa Catarina & 28 & 5.1 \\
Santiago & 2 & 0.4 \\
San Pedro Garza García & 4 & 0.7 \\
Total & 547 & 100 \\
\hline Vivienda & Frecuencia & Porcentaje \\
\hline Casa unifamiliar & 444 & 81.2 \\
Casa multifamiliar & 65 & 11.9 \\
Departamento & 31 & 5.7 \\
Otro & 7 & 1.3 \\
Total & 547 & 100 \\
\hline
\end{tabular}

Fuente: Elaboración propia

Para que se hable de que el instrumento es idóneo, y que se pueda utilizar con toda la confianza se requiere que cumpla con dos requisitos: confiabilidad y validez (González, 2008). El instrumento de medida que utilizamos para demostrar que las muestra con la que trabajamos posee validez y fiabilidad es el alfa de Cronbach. El Coeficiente Alfa de Cronbach, requiere una sola administración del instrumento de medición y produce valores que oscilan entre 0 y 1 . Su ventaja reside en que no es necesario dividir en dos mitades a los ítems del instrumento de medición, simplemente se aplica la medición y se calcula el coeficiente" (Hernández, 2003). En este artículo se calculó mediante el software Statistical Package for the Social Sciences (SPSS). El coeficiente de alfa de Cronbach se encuentra en un treinta y uno por ciento $(31 \%)$ que, según Palella, S. y Martins, F. (2003) el coeficiente es bajo.

Sin embargo, el alfa de Cronbach tiene una mayor efectividad en preguntas con resolución basada en una escala Likert, el alfa puede no otorgar los resultados deseados al implementar un instrumento con escalas diferentes, como es el caso de las variables binarias, para ello existen otros estadísticos que pueden sustentar los resultados obtenidos. El alfa de Cronbach no es una consistencia interna. Tampoco es una medición del grado de unidimensionalidad. El alfa ha sido empleada para correlacionarse con muchos otros estadísticos y muchos de estos resultados han sido interesantes, también han sido confusos en el sentido que, sin información adicional, tanto valores de alfa muy altos como muy bajos pueden ir con unidimensionalidad o multidimensionalidad de los datos. Pero dado que se necesita información adicional para saber qué sustenta, el alfa de Cronbach por sí mismo no puede ser interpretado como una medida de consistencia interna (Sijtsma, 2009).

En el instrumento realizado se muestran los lugares más visitados fuera de casa a raíz de la contingencia por COVID-19 en la zona metropolitana de Monterrey. De esta manera se permite apreciar aquellos espacios en los cuales no se ha podido adaptar la vivienda y la población se ha visto en la necesidad de salir a ellos. Las áreas de comercios y servicios como bancos, farmacias y tiendas de conveniencia, son los espacios que la muestra expresó visitar mayormente hoy en día con una frecuencia de 235 encuestados, el $43.0 \%$ de la muestra, mientras que en segundo puesto se encontró al trabajo con una frecuencia de 157 encuestados que representa el $28.7 \%$ de la muestra.

Dichos datos nos muestran que las restricciones impuestas por los organismos gubernamentales lograron el objetivo de reducir las visitas a espacios que no son considerados como de primera necesidad, siendo los espacios de trabajo, para aquellos que no lograron adaptarlo a un espacio dentro de casa y claramente los espacios de comercios y servicios donde la población 
necesita abastecerse de los productos y servicios brindados por estos sectores.

En la Ilustración 1 se muestra la relación existente entre los diferentes municipios de la zona metropolitana de Monterrey donde habita la población encuestada y el nivel de adaptación que perciben los sujetos de estudio con respecto a su vivienda. El $26.69 \%$ de la muestra habita en Monterrey, en segundo lugar, con $23.03 \%$ el municipio de San Nicolás y en último lugar con $0.36 \%$ de la muestra cada uno los municipios de Cadereyta Jiménez, Salinas Victoria y Santiago.

Los habitantes de los municipios de
Monterrey, Santiago y San Pedro Garza García perciben tener una vivienda con el nivel máximo de adaptación plasmado en el instrumento, seguido por los municipios de General Escobedo, Juárez y San Nicolás de los Garza, quienes consideran suficiente el nivel en que sus viviendas están adaptadas ante el confinamiento. El resto de los encuestados de los otros municipios que forman parte de la zona metropolitana de Monterrey percibe una adaptación neutra, a excepción del municipio de Salinas Victoria en donde los únicos dos encuestados participantes perciben un poco nivel de adaptación de su vivienda ante el confinamiento.

Gráfica 1. Relación entre la percepción de la adaptación de la vivienda y el municipio donde se habita.

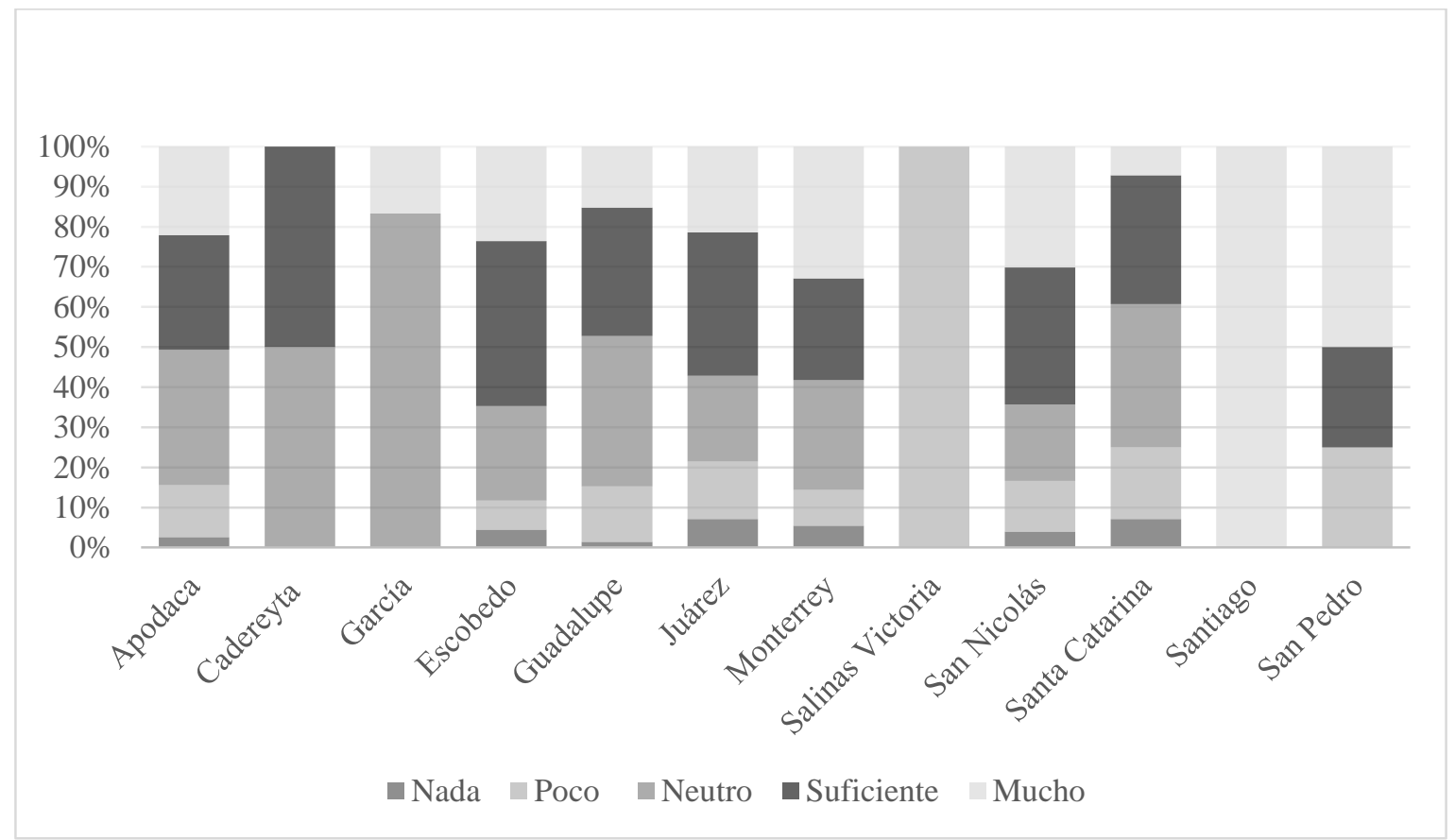

Fuente: Elaboración propia

El conocimiento sobre casos de COVID-19 y el número de habitantes con los que se comparte la vivienda resulta ser una relación de variables que influye en la percepción de la adaptación personal ante la contingencia. En la Ilustración 2 la variable COVID-19 tuvo una clasificación en cuatro niveles, el primero con respuesta de aquellos que padecieron o que aún padecen la enfermedad, en segundo aquellos que padecen o padecieron la enfermedad y además conocen a alguien que también la padece o padeció, en tercero aquellos que solo conocen a alguien enfermo o que enfermó y por último aquellos encuestados que no tienen conocimiento alguno de haber enfermado o conocer alguien que enfermara o esté enfermo por COVID-19.

El $80.80 \%$ de los encuestados expresaron conocer a alguien enfermo o que enfermó, además de que consideran estar suficientemente adaptados en función de la cantidad de personas con las que comparten la 
vivienda, teniendo una media de 4 habitantes por vivienda. Los encuestados con tendencia a compartir con mayor número de habitantes perciben un nivel neutro de adaptación personal ante el confinamiento en casa. En el primer nivel, los encuestados que expresaron estar enfermos o haberlo estado tuvieron un nivel de adaptación neutro, de igual manera que aquellos que están o han estado enfermos y que además conocen a alguien que está o estuvo enfermo.

Por otra parte, el grupo de encuestados que percibe el nivel de adaptación personal máximo fue en el de aquellos sujetos de estudio que expresaron no tener conocimiento alguno de haber enfermado o conocer a alguien enfermo, representando tan solo el $2.74 \%$ de la muestra total del instrumento.

Gráfica 2. Relación del conocimiento de casos de COVID-19 y la percepción de adaptación personal por contingencia

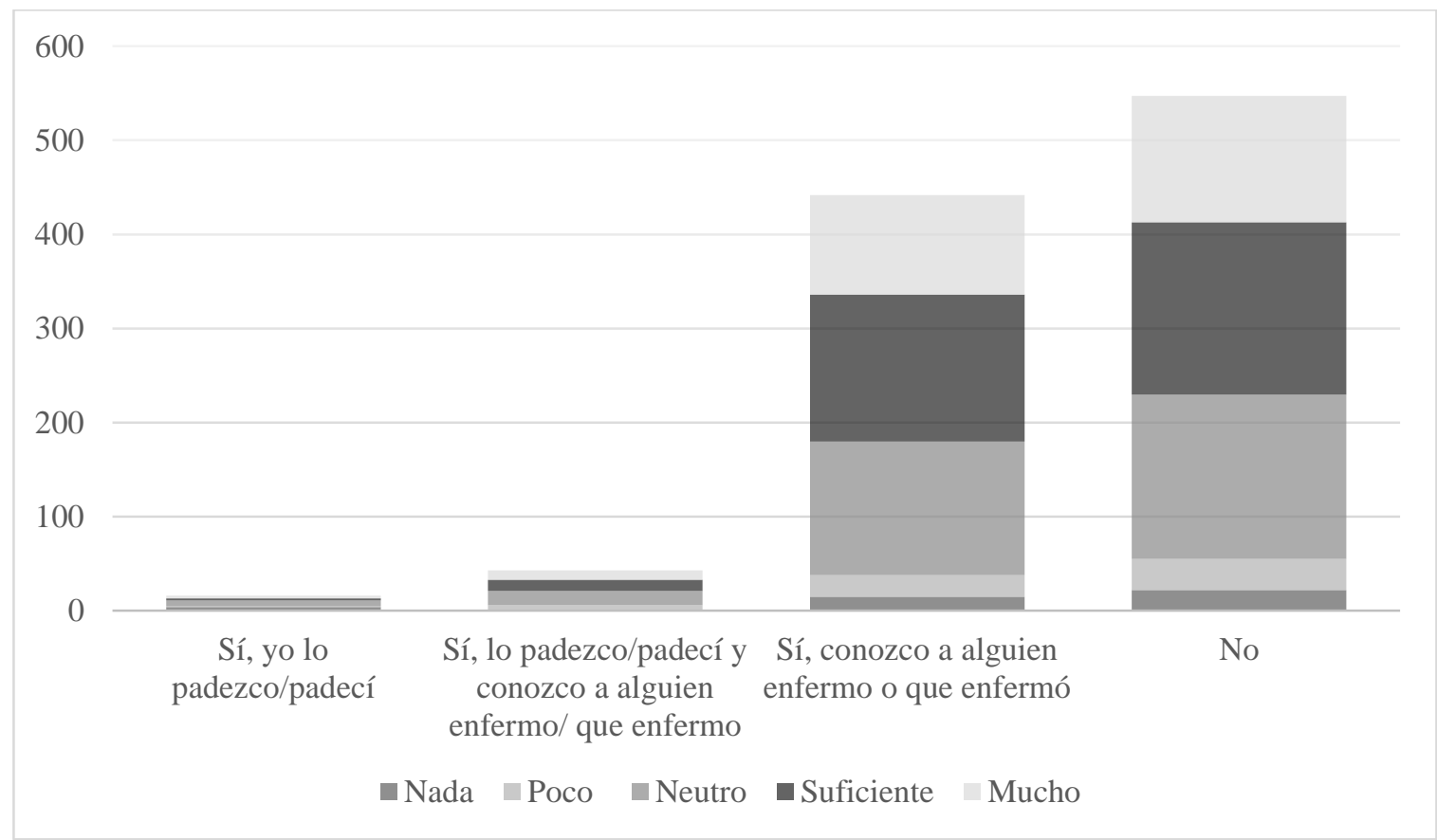

Fuente: Elaboración propia

En la Tabla 4 se presentan los espacios de la vivienda y cuáles de ellos han sido más versátiles al momento de que la población ha tenido la necesidad de adaptarlos para actividades que no son propiamente del espacio, todo ello en función de la tipología de vivienda, casa unifamiliar, casa multifamiliar, departamento y otro sin especificar. Los espacios de la vivienda, interiores sociales como la sala, el comedor, la cocina, los interiores privados como la recámara y los espacios exteriores tales como la cochera, el jardín, patio, balcón y terraza.
El espacio que los encuestados más han adaptado para actividades de trabajo es en primer lugar la recámara con una frecuencia de 229 en la casa unifamiliar, 40 en la casa multifamiliar, 13 en el departamento y 1 en una tipología de vivienda no especificada, 283 encuestados de la muestra optó por este espacio. El espacio más adaptado para actividades de ocio resultó ser la recámara con una frecuencia de 97 sujetos de estudios en casa unifamiliar, 23 en casa multifamiliar y 11 en departamento, es decir 131 de los 547 encuestados expresaron este resultado. 
Los espacios mayormente percibidos como favoritos dentro de la muestra fueron los espacios interiores privados, con una frecuencia de 220, siendo la recámara el espacio más seleccionado en todas las tipologías de vivienda. Lo que nos permite deducir que el espacio que resultó ser más versátil y adaptable es la recámara, puesto que fue el espacio más utilizado para realizar actividades de trabajo, de ocio y también el espacio favorito de los encuestados, al ser tan flexible ha permitido que la muestra se identifica mayormente con esta área de la vivienda.

Tabla 4. Espacios más adaptables de trabajo, ocio y el espacio favorito de los encuestados en las distintas tipologías de vivienda.

\begin{tabular}{|c|c|c|c|c|c|}
\hline \multirow[b]{2}{*}{ Espacio_trabajo } & \multicolumn{4}{|c|}{ Vivienda } & \multirow[b]{2}{*}{ Total } \\
\hline & $\begin{array}{c}\text { Casa } \\
\text { unifamiliar }\end{array}$ & $\begin{array}{c}\text { Casa } \\
\text { multifamiliar }\end{array}$ & Departamento & Otro & \\
\hline Sala & 71 & 8 & 5 & 0 & 84 \\
\hline Comedor & 71 & 9 & 8 & 3 & 91 \\
\hline Cocina & 4 & 0 & 0 & 0 & 4 \\
\hline Recámara & 229 & 40 & 13 & 1 & 283 \\
\hline $\begin{array}{l}\text { Espacios exteriores (patio, } \\
\text { terraza, balcón o cochera, etc.) }\end{array}$ & 16 & 1 & 0 & 0 & 17 \\
\hline $\begin{array}{l}\text { No realicé trabajo/ escuela en } \\
\text { casa }\end{array}$ & 53 & 7 & 5 & 3 & 68 \\
\hline Total & 444 & 65 & 31 & 7 & 547 \\
\hline \multicolumn{6}{|l|}{ Espacio_ocio } \\
\hline Sala & 81 & 11 & 5 & 1 & 98 \\
\hline Comedor & 9 & 0 & 0 & 0 & 9 \\
\hline No realicé adecuaciones & 203 & 23 & 9 & 6 & 241 \\
\hline Cocina & 5 & 0 & 1 & 0 & 6 \\
\hline Recámara & 97 & 23 & 11 & 0 & 131 \\
\hline $\begin{array}{l}\text { Espacios exteriores (patio, } \\
\text { terraza, balcón, cochera) }\end{array}$ & 49 & 8 & 5 & 0 & 62 \\
\hline Total & 444 & 65 & 31 & 7 & 547 \\
\hline \multicolumn{6}{|l|}{ Espacio_favorito } \\
\hline $\begin{array}{l}\text { Espacios exteriores (cochera, } \\
\text { jardín, terraza, balcón, etc.) }\end{array}$ & 96 & 17 & 6 & 2 & 121 \\
\hline $\begin{array}{l}\text { Espacios interiores de trabajo/ } \\
\text { escuela }\end{array}$ & 27 & 2 & 2 & 0 & 31 \\
\hline $\begin{array}{l}\text { Espacios interiores recreativos } \\
\text { (gimnasio en casa, oratorio, etc.) }\end{array}$ & 13 & 3 & 2 & 0 & 18 \\
\hline $\begin{array}{l}\text { Espacios interiores sociales } \\
\text { (comedor, cocina o sala) }\end{array}$ & 129 & 19 & 8 & 1 & 157 \\
\hline $\begin{array}{l}\text { Espacios interiores privados } \\
\text { (recámara) }\end{array}$ & 179 & 24 & 13 & 4 & 220 \\
\hline Total & 444 & 65 & 31 & 7 & 547 \\
\hline
\end{tabular}

Fuente: Elaboración propia

En la tabla 5 se detalla el tipo de adecuaciones que se realizaron en los espacios que los encuestados emplearon para uso de trabajo, de ocio y espacios exteriores, conforme al género con el que se identifican los sujetos de estudio. Las adecuaciones fueron divididas en cambios estéticos, cambios constructivos, en la adquisición y colocación de vegetación y en la 
opción de la no realización de adecuaciones. En las adecuaciones destinadas al espacio adaptado al trabajo, el $34.95 \%$ de género femenino y el $41.41 \%$ de género masculino expresó realizar cambios estéticos siendo estos los mayormente efectuados. En las adecuaciones al espacio de ocio nuevamente los cambios estéticos fueron los dominantes con $30.08 \%$ del género femenino y $34.34 \%$ del género masculino. Por último, en las adecuaciones realizadas en los espacios exteriores de la vivienda, los cambios más efectuados por el género femenino con un $17.48 \%$ fueron los de adquirir y plantar vegetación y los cambios más efectuados por el género masculino con un $15.15 \%$ fueron los cambios estéticos.

Los resultados obtenidos expresan que a pesar de que los cambios estéticos fueron las adecuaciones mayormente realizadas, más de la mitad de la población mostró no haber realizado adecuación alguna en los espacios de la vivienda. El $59.41 \%$ en el espacio de trabajo, $65.63 \%$ el espacio de ocio y $60.32 \%$ en los espacios exteriores, infiriendo que los encuestados encontraron formas de darle más de un uso a los espacios preexistentes a la contingencia por COVID-19, mostrando versatilidad en las viviendas y obteniendo espacios multifuncionales.

Tabla 5. Tipos de adecuaciones realizadas según el género de los encuestados.

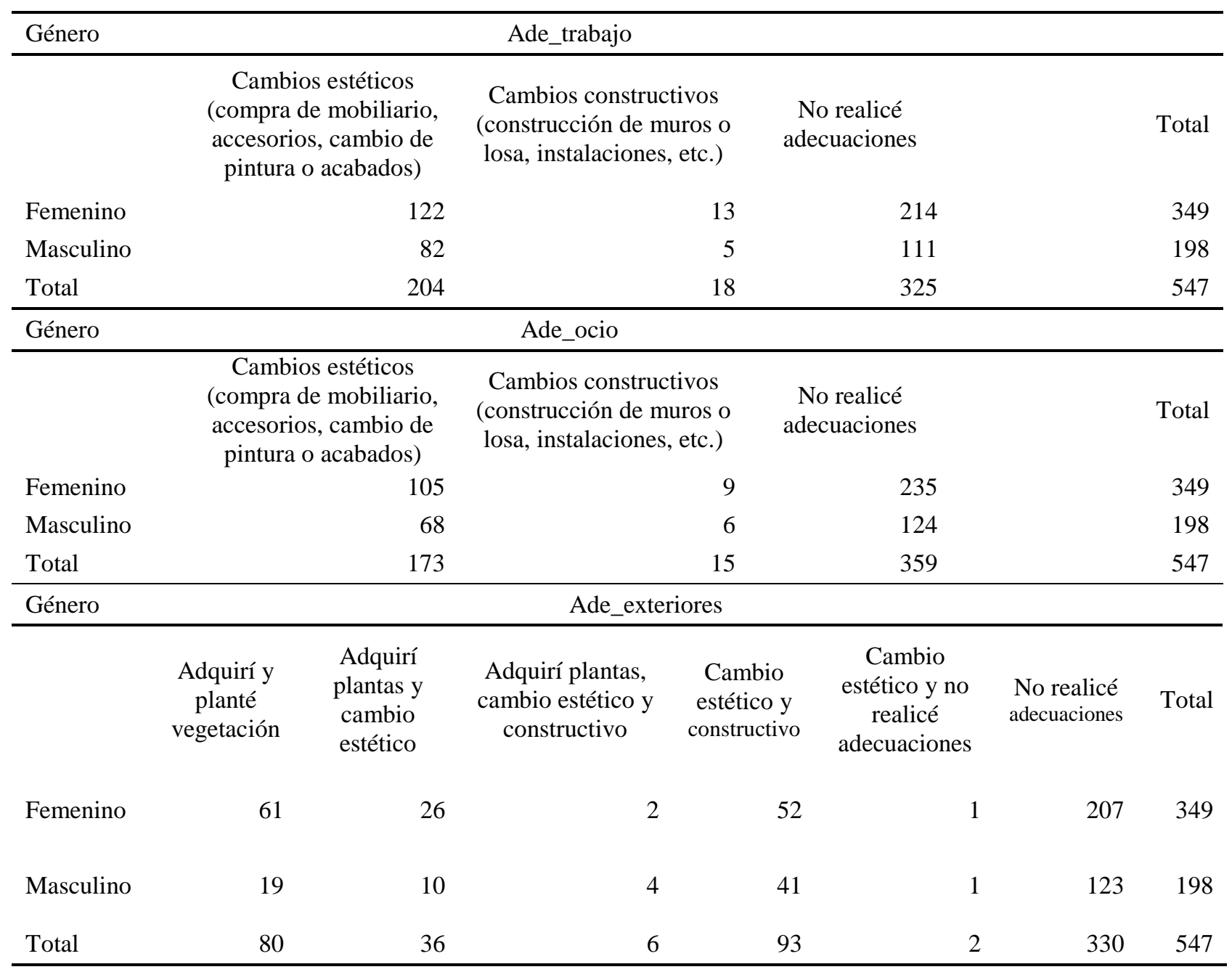

Fuente: Elaboración propia

En la tabla correlación de variables (véase

anexo 1) los resultados obtenidos del método 
de correlación de Pearson que se utilizó para evaluar la confiabilidad de cada variable del instrumento de medición. Para un conjunto de datos, el valor $r$ de este coeficiente puede tomar cualquier valor entre $1 \mathrm{y}+1$. El valor de $r$ es positivo si existe una relación directa entre ambas variables, esto es, si las dos aumentan al mismo tiempo. Será negativo si la relación es inversa, es decir, cuando una variable disminuye a medida que la otra aumenta. Un valor de +1 o -1 indica una relación lineal perfecta entre ambas variables, mientras que un valor 0 indica que no existe relación lineal entre ellas (Pértegas, 2002). Para esta investigación se aceptaron sólo aquellos con resultados distintos a 0.00 pero que oscilan entre el +1 y -1 y con una significancia bilateral de $5 \%$ marcado como * $\mathrm{p}<0.05$ y aquellas con una significancia bilateral de $1 \%$ con $* * \mathrm{p}<0.01$.

Las características individuales sociodemográficas como son el género y las características de la vivienda como la tipología, número de habitantes que comparten la vivienda y el municipio en el que residen durante el confinamiento son variables usadas como control de la muestra que nos permiten conocer la percepción sobre el nivel de adaptación en sus viviendas y las adecuaciones realizadas ante el confinamiento de los habitantes de la zona metropolitana de Monterrey. A continuación, se describirá la interpretación de cada una de las variables que resultaron estadísticamente significativas.

La percepción de adaptación de la vivienda, es decir el nivel en que una persona considera que su vivienda está preparada para el confinamiento, está fuertemente correlacionada con la cantidad de personas con las que comparte la vivienda, con un coeficiente de correlación de -.110 y esta última variable es también estadísticamente significativa con el municipio donde dichos habitantes residen, con un coeficiente de Pearson de -.107. Estos resultados indican que los municipios donde se encontró una media de 4 habitantes por vivienda percibieron una mayor adaptación de esta ante el confinamiento, siendo éstos los municipios de Monterrey, Santiago y San Pedro Garza García. Los resultados señalan que los habitantes tienen una percepción de adaptación personal ante la vida en confinamiento y está fuertemente correlacionada con el conocimiento de casos de COVID-19 en su entorno, aquellos que expresaron conocimiento de casos indicaron estar suficientemente adaptados al confinamiento mientras que aquellos que expresaron desconocimiento de casos indicaron estar mucho más adaptados, estas variables presentan un coeficiente de correlación de .107. Además de que la adaptación personal es estadísticamente significativa con una correlación negativa a la cantidad de habitantes que residen por vivienda, con un coeficiente de Pearson del .102. La percepción en adaptación personal se ve directamente influenciada por la cantidad de personas con que se comparte la vivienda, siendo la media de cuatro personas con un nivel de neutralidad en la adaptación.

La variable de espacio de trabajo posee una correlación estadísticamente significativa con las variables de espacio de ocio, con una correlación de Pearson de .195 y con la variable espacio favorito de .087. Estos resultados nos indican que siendo la recámara el espacio favorito del $40.22 \%$ de la muestra optan por usar dicho espacio en sus actividades de trabajo, con un $51.73 \%$ y de ocio, con un $23.94 \%$ de los encuestados.

La variable de adecuación en el espacio de trabajo y adecuación en el espacio de ocio están fuertemente correlacionadas con un coeficiente de Pearson del .443. Los resultados muestran que al ser la recámara el espacio predilecto de la población encuestada es el lugar de trabajo y ocio aquellos que experimentan los mayores cambios estéticos, evidenciando la versatilidad y multifuncionalidad de la recámara en la vivienda.

\section{CONCLUSIONES}

En este artículo se realizó un análisis descriptivo del instrumento efectuado a una muestra de 547 habitantes de la zona metropolitana de Monterrey, donde los encuestados señalaron su percepción de adaptación personal y de sus viviendas ante el nuevo estilo de vida en confinamiento por 
la pandemia de COVID-19.

Entre los principales hallazgos se encontró que la media de habitantes por vivienda en la zona metropolitana de Monterrey es de cuatro habitantes ocupando en su mayoría la tipología de vivienda de casa unifamiliar. Los habitantes del municipio de Monterrey fueron los que perciben una mayor adaptación de sus viviendas en confinamiento, seguido por los municipios colindantes del sur, mientras que el resto mostraron menor adaptación a mayor distanciamiento de la capital, Salinas Victoria, siendo el municipio más alejado presentó un nivel clasificado como poco, siendo el nivel más bajo registrado en la muestra. El segundo de los hallazgos fue que los encuestados con menor conocimiento de casos de COVID-19, fueron aquellos que tienen una percepción de adaptación personal mayor, mientras que las personas que padecen o padecieron la enfermedad fueron los menos adaptados con un nivel neutro. En tercer lugar, el instrumento identificó a la recámara como el espacio predilecto por los encuestados, siendo un espacio interior y privado, además, fue el espacio más multifuncional en las distintas tipologías de vivienda, coronándose como el espacio más utilizado para realizar actividades de trabajo y ocio. Los sujetos de estudio identificados con el género masculino realizaron más cambios estéticos al interior de la vivienda, mientras que los identificados con el género femenino realizaron mayores cambios en la adquisición y planta de vegetación en los espacios exteriores de la vivienda.

Es de importancia señalar que somos del conocimiento que el instrumento pudiese mejorar al emplear un muestreo de carácter probabilístico, sin embargo, comenzando por este artículo para ser promotor de futuros proyectos de investigación que se centren en el análisis de la importancia de la vivienda para la sociedad, el impacto en modo de vida de los habitantes y los espacios que la conforman ante situaciones de gran índole en los ámbitos social, político, económico y como lo es en este momento, en el sector salud con el COVID-19 


\section{REFERENCIAS}

Bisquerra, R. (1989). Introducción conceptual al análisis multivariable. Un enfoque informático con los paquetes SPSS-X, BMDP, LISREL y SPAD. Barcelona: PPU.

García, M. (1992). El análisis de la realidad social: métodos y técnicas de investigación. Madrid: Alianza Universidad.

González, Y. (2008). Instrumento Cuidado de comportamiento profesional: validez y confiabilidad, vol. 8, no. 2, Chia, Colombia, pp. 170-182.

Hernández, R. (2003). Metodología de la Investigación, Editorial Mc. Graw Hill, México D.F.

Hernández, J., \& Paternostro, R. (2020). Diseño de vivienda para afrontar una pandemia. Recuperado el 25 de Marzo de 2021, de https://cutt.ly/Ebne3Z1

Instituto Nacional de Estadística, Geografía e Informática. (16 de Marzo de 2021). Presentación de resultados Censo 2020 Nuevo León. Obtenido de https://cutt.ly/KndWVaL

Moreno, R. y Alvarado, E. (2011). El entorno social y su impacto en el precio de la vivienda: un análisis de precios hedónicos en el área metropolitana de Monterrey. Trayectorias, 14(33), 131-147.

Murray, R., \& Larry, J. (2009). Estadística. Mexico, D.F.: McGraw-Hill.

ONU-Habitat. (2021). Cities and Pandemies Towards a More Just Green and Healthy Future. [Ciudades y pandemias hacia un futuro más justo, verde y saludable]. UN-Habitat. vol. 1, no.1, 1-194.

ONU-Habitat . (30 de Marzo de 2021). Informe Ciudades y Pandemias: Hacia un futuro más justo, verde y equitativo. Obtenido de https://cutt.ly/Vbnrypu

Organización Mundial de la Salud. (24 de Febrero de 2010). Alerta y Respuesta Mundiales (GAR). Obtenido de ¿Qué es una pandemia?: https://cutt.ly/WbnrssP

Organización Mundial de la Salud. (11 de marzo de 2020). Alocución de apertura del Director General de la OMS en la rueda de prensa sobre la COVID-19 celebrada el 11 de marzo de 2020. [Consultado el 24 de octubre de 2020]. Disponible en: https://www.who.int/es/dg/speeches

Organización Mundial de la Salud. (25 Marzo de 2020). Coronavirus disease 2019 (COVID-19) Situation Report - 65. [Informa de situación de la enfermedad por Coronavirus 2019 (COVID19) ]. 1-12.

Organización Mundial de la Salud. (2 Marzo de 2021). Weekly epidemiological update - 2 March 2021. [Actualización semanal de marzo 2021]. Obtenido de: https://cutt.ly/4bnrg9A

Palella, S. y Martins, F. (2003). Metodología de la Investigación cuantitativa. Caracas: Fedupel.

Pérez-Pérez, A. (30 de Julio de 2015). El diseño de la vivienda de interés social. La satisfacción de las necesidades y expectativas del usuario. Revista de Arquitectura, 18(1). DOI:10.14718/RevArq.2016.18.1.7.

Pita Fernández S., Pértega Díaz S. (2002). Determinación del tamaño muestral para calcular la significación del coeficiente de correlación lineal . Unidad de Epidemiología Clínica y Bioestadística. Complexo Hospitalario Juan Canalejo. 209-211.

Programa de las Naciones Unidas para los Asentamientos Humanos ONU-Habitat. (2018). Vivienda y ODS en México. [Consultado el 30 de noviembre de 2020]. Disponible en: http://70.35.196.242/onuhabitatmexico/V

Sijtsma, K. (2009). On the use, the misuse, and the very limited usefulness of Cronbach's alpha. [Sobre el uso, el mal uso y la utilidad limitada del alfa de Cronbach]. Psychometrika. vol.74, no. $1,107-120$.

Universidad Politécnica de Madrid. (Junio de 2020). La mitigación del impacto del COVID-19 en contextos de precariedad. Posibles medidas desde la perspectiva de la habitabilidad básica. Obtenido de https://cutt.ly/0bneZTr 


\section{ANEXOS}

Anexo 1. Correlación de variables.

Correlación de variables de gráfica 1

\begin{tabular}{|c|c|c|c|c|c|}
\hline \multirow[t]{3}{*}{ Habitantes } & Correlación de Pearson & $\begin{array}{l}\text { Municipio } \\
-.107^{*}\end{array}$ & $\begin{array}{r}\text { Adap_vivienda } \\
-.110^{*}\end{array}$ & \multicolumn{2}{|c|}{ Habitantes } \\
\hline & Sig. (bilateral) & 0.013 & 0.010 & & \\
\hline & $\mathrm{N}$ & 547 & 547 & & 547 \\
\hline \multicolumn{6}{|c|}{ Correlación de variables de gráfica 2} \\
\hline \multirow[t]{3}{*}{ Adap_Personal } & Correlación de Pearson & $\begin{array}{r}\text { COVID-19 } \\
.107^{*}\end{array}$ & $\begin{array}{r}\text { Habitantes } \\
-.102^{*}\end{array}$ & \multicolumn{2}{|c|}{ Adap_personal } \\
\hline & Sig. (bilateral) & 0.012 & 0.017 & & \\
\hline & $\mathrm{N}$ & 547 & 547 & & 547 \\
\hline \multicolumn{6}{|c|}{ Correlación de variables de tabla 4} \\
\hline \multirow[t]{3}{*}{ Espacio_trabajo } & Correlación de Pearson & $\begin{array}{r}\text { Vivienda } \\
0.016\end{array}$ & $\begin{array}{r}\text { Espacio_trabajo } \\
1\end{array}$ & $\begin{array}{r}\text { Espacio_ocio } \\
.195^{* *}\end{array}$ & $\begin{array}{l}\text { Espacio } \\
\text { favorito } \\
\qquad .087^{*}\end{array}$ \\
\hline & Sig. (bilateral) & 0.715 & & 0.000 & 0.042 \\
\hline & $\mathrm{N}$ & 547 & 547 & 547 & 547 \\
\hline \multicolumn{6}{|c|}{ Correlación de variables de tabla 5} \\
\hline \multirow[t]{3}{*}{ Ade_trabajo } & Correlación de Pearson & $\begin{array}{l}\text { Género } \\
-0.059\end{array}$ & Ade_trabajo & Ade & $.443^{* *}$ \\
\hline & Sig. (bilateral) & 0.170 & & & 0.000 \\
\hline & $\mathrm{N}$ & 547 & 547 & & 547 \\
\hline
\end{tabular}

